\title{
A novel Zigbee-based data acquisition system for distributed photovoltaic generation in smart grid
}

\begin{abstract}
Distributed photovoltaic (PV) power plant monitoring is one of the crucial aspects for energy forecasting and demand management in the future smart grid (SG) through establishing lowpowered communication technology. In this paper, Zigbee wireless technology is considered for AC electrical parameters' monitoring of the distributed PV plant located at Universiti Putra Malaysia (UPM). For this consideration, relevant measurement circuits, Arduino UNO embedded board, and Zigbee radio are interfaced to create a wireless (WS)-node for installing in the PV inverter. In addition, a LabVIEW program is implemented and linked to Microsoft Access Database (MS Access DB) at the control center server system for the Zigbee-based wireless data acquisition, storage, and monitoring. The obtained data from the developed system are also validated with the actual PV generation. The results are found comparable and it also reveals that low-powered Zigbee is ideal for monitoring the distributed PV generation where the data delivery requirement is not urgent.
\end{abstract}

Keyword: Database; Photovoltaic module; Smart grid; Wireless network; Zigbee 